\title{
Search for cascade events with Baikal-GVD demonstration cluster "Dubna"
}

\author{
A.D. Avrorin ${ }^{a}$, A.V. Avrorin ${ }^{a}$, V.M. Aynutdinova, R. Bannash ${ }^{g}$, I.A. Belolaptikov ${ }^{b}$, \\ V.B. Brudanin ${ }^{b}$, N.M. Budnev" ${ }^{\text {, I.A. Danilchenkoa }}$, G.V. Domogatskya , A.A. \\ Doroshenko $^{a}$, R. Dvornickýb,h, A.N. Dyachok ${ }^{c}$, Zh.-A.M. Dzhilkibaev ${ }^{a}$, L. Fajt ${ }^{b, h, i}$, \\ S.V. Fialkovskye, A.R. Gafarov', K.V. Golubkova, T.I. Gress ${ }^{c}$, Z. Hons ${ }^{b}$, K.G.

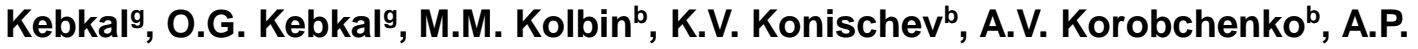 \\ Koshechkin $^{\text {a }}$, F.K. Koshel ${ }^{\text {a }}$, A.V. Kozhin ${ }^{\text {d, V.F. Kulepov', D.A. Kuleshov }}{ }^{\text {a }}$, M.B. \\ Milenin $^{\text {e, R.A. Mirgazov', E.R. Osipovad, A.I. Panfilov }}{ }^{\mathrm{a}}$, L.V. Pan'kov ${ }^{c}$, D.P. \\ Petukhov $^{a}$, E.N. Pliskovsky, M.I. Rozanov ${ }^{f}$, E.V. Rjabov ${ }^{c}$, G.B. Safronov ${ }^{b}$, B.A. \\ Shaybonov $^{* b}$, M.D. Shelepov ${ }^{a}$, F. Simkovic ${ }^{b, h, i}$, A.V. Skurikhin ${ }^{d}$, I. Stekli, O.V. \\ Suvorova ${ }^{a}$, V.A. Tabolenkoc, B.A. Tarashanskyc, S.A. Yakovlev ${ }^{g}$, A.V. \\ Zagorodnikov $^{\mathrm{c}}$ and V.L. Zurbanov ${ }^{\mathrm{c}}$ \\ ${ }^{a}$ Institute for Nuclear Research, Moscow, 117312 Russia \\ ${ }^{b}$ Joint Institute for Nuclear Research, Dubna, 141980 Russia \\ ${ }^{c}$ Irkutsk State University, Irkutsk, 664003 Russia \\ ${ }^{d}$ Institute of Nuclear Physics, Moscow State University, Moscow, 119991 Russia \\ eNizhni Novgorod State Technical University, Nizhni Novgorod, 603950 Russia \\ ${ }^{f}$ St. Petersburg State Marine Technical University, St. Petersburg, 190008 Russia \\ ${ }^{g}$ EvoLogics, Germany \\ ${ }^{h}$ Comenius University, Bratislava, Slovakia \\ ${ }^{i}$ Czech Technical University in Prague, Prague, Czech Republic \\ E-mail: bairsheyandex.ru
}

Deep underwater neutrino telescope „Dubna“ - the first, demonstration cluster of Baikal-GVD, has been deployed in April 2015 and was operating up to February 2016 in Lake Baikal. In 2016 this array was upgraded to baseline configuration of GVD-cluster by adding additional 96 optical modules. The next step of array extension was provided in 2017 by deployment of second GVD-cluster in Lake Baikal. We present here preliminary results of a search for cascade events using data sample recorded in 2015 by Dubna array and corresponding to 41 live days of data taking. 


\section{Introduction}

Deep underwater neutrino telescope Baikal Gigaton Volume Detector (GVD) is currently under construction in Lake Baikal [1]. The Baikal-GVD is formed by a three-dimensional lattice of optical modules those are photomultiplier tubes housed in transparent pressure spheres, arranged at vertical load-carrying cables to form strings. The telescope has a modular structure and consists of functionally independent clusters - sub-arrays comprising 8 strings of OMs, which are connected to shore by individual electro-optical cables. The first, reduced size cluster named "Dubna" has been deployed and operated during 2015 in Lake Baikal. In April 2016, this array has been upgraded to baseline configuration of GVD-cluster, which comprises 288 optical modules attached at 8 strings at depths from $750 \mathrm{~m}$ to $1275 \mathrm{~m}$. In April 2017 second GVD-cluster was commissioned in Lake Baikal, increasing the total number of operated optical modules up to 576 OMs. During Phase-1 of Baikal-GVD implementation an array consisting of eight clusters will be deployed by 2020. Since each GVD-cluster represents a multi-megaton scale Cherenkov detector, studies of neutrinos of different origin are allowed at early stage of Baikal-GVD construction. Recently, the high-energy astrophysical neutrino signal has been reported by IceCube [2]. Data sample of high-energy starting event (HESE) analysis comprises 54 events with a reconstructed energy above $30 \mathrm{TeV}, 39$ of which are identified as cascades and 14 as track events [3]. These results demonstrate an efficiency of cascade mode of neutrino detection by neutrino telescopes. Baikal Collaboration has a long-term experience to search for diffuse neutrino flux with NT200 array using cascade mode [4,5]. The array "Dubna" has the potential to record astrophysical neutrinos with a flux values measured by IceCube [6]. A search for high-energy neutrinos with "Dubna" array is based on the selection of cascade events generated by neutrino interactions in the sensitive volume of array. Here we describe the cascade event simulation and reconstruction procedures and discuss the first preliminary results obtained by analysis of data collected with "Dubna" array in 2015.

\section{Simulation and reconstruction of cascades}

\subsection{Cherenkov radiation of cascades}

The total number of Cherenkov photons from an electromagnetic or hardronic cascade is proportional to the cascade energy $E_{\mathrm{sh}}$ :

$$
N_{\text {tot }}^{c h}=B E_{\text {sh }}, \quad B=(1.04 \div 1.16) 10^{5} \mathrm{GeV}^{-1}, \text { for electromagnetic showers. }
$$

In a case of hadronic showers:

$$
\begin{gathered}
B=0.86 \cdot 625 \cdot f(\epsilon) n_{c h} \mathrm{GeV}^{-1}, \\
f(\varepsilon)=-1.27 \cdot 10^{-2}-4.76 \cdot 10^{-2}(\varepsilon+3)-2.07 \cdot 10^{-3}(\varepsilon+3)^{2}+0.52 \sqrt{\varepsilon+3}, \\
\varepsilon=\log _{10}\left(E_{\text {sh }} / 1 \mathrm{TeV}\right),
\end{gathered}
$$

where $n_{c h}$ is a linear density of Cherenkov radiation of electrons. The number of Cherenkov photons $N_{c h}(x, \theta, t) d x d \Omega d t$ emitted at time $t$ from an interval $d x$ along the shower near point $x$ in a spatial angle $d \Omega$ at an angle $\theta$ to the shower axis can be represented as:

$$
N_{c h}(x, \theta, t) d x d \Omega d t \cong N_{e^{ \pm}}(x) \Psi_{c h}(\theta) n_{c h} \delta(t-x / c) d x d \Omega d t
$$

where $N_{e} \pm(x)$ is a linear density of electrons and positrons along the shower, $\Psi_{c h}(\theta)$ is the angular distribution of Cherenkov photons. For electromagnetic showers, the following approximation has been used: 


$$
\begin{gathered}
N_{e^{ \pm}}(x)=\left(\frac{E_{s h}}{E_{c}}\right)^{S} S^{-1.5 X} \exp (-2.49 S+0.5(S-1) X+0.025), \\
S=\frac{3 X}{X+2 \ln \left(E_{s h} / E_{c}\right)-2.4}, \quad E_{c}=72 \mathrm{MeV}, X=x / X_{0}, X_{0}=36.1 \mathrm{~cm} .
\end{gathered}
$$

For hadronic showers:

$$
N_{e^{ \pm}}(x)=S \frac{E_{s h}}{E_{c}}\left(1-\frac{\lambda}{X_{\max }}\right) \exp \left(\frac{X_{\max }-x}{\lambda}-1\right)\left(\frac{x}{X_{\max }-\lambda}\right)^{X_{\max } / \lambda},
$$

$X_{\text {max }}=X_{r} \ln \left(E_{s h} / E_{c}\right), \quad S=0.11842, \quad X_{r}=39.502 \mathrm{~cm}, \lambda=113.03 \mathrm{~cm}, \quad E_{c}=0.17 \mathrm{GeV}$.

Photons emitted from vicinity of shower profile maximum dominate in a total Cherenkov radiation. It allows to use the angular distribution of Cherenkov photons in the shower maximum $\Psi_{\mathrm{ch}}(\theta)$ (see, Fg.1) for every shower interval $\Delta x$. The number of Cherenkov photons emitted from an interval $\Delta x$ along the shower near $x$ can be represented as:

$$
N_{c h}(x, \Delta x, \theta, t)=B E_{0} \Psi_{c h}(\theta) \delta(t-x / c) \int_{x-\Delta x}^{x+\Delta x} \widetilde{N}_{e^{ \pm}}\left(x^{\prime}\right) d x^{\prime},
$$

where $\widetilde{N}_{e^{ \pm}}(x)$ is the normalized linear density of $e^{ \pm}$along a shower. The algorithm for simulating the generation of Cherenkov radiation from high-energy showers appears as follows. The longitudinal shower length is divided into intervals $\Delta x$ and the shower is considered as a superposition of point sources of Cherenkov radiation located at the center of each interval $\Delta x$ with the intensity $N_{c h}(\mathrm{x}, \Delta \mathrm{x}, \theta, t)$ derived from (1).

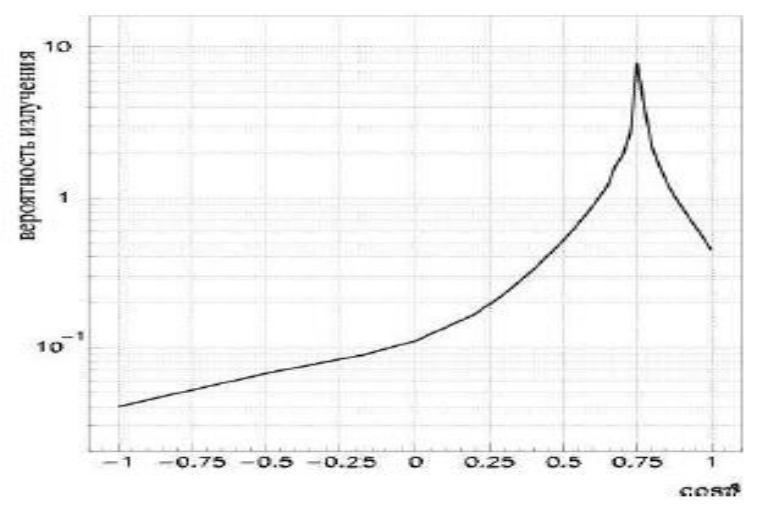

Fig. 1: The angular distribution of Cherenkov radiation from high-energy electromagnetic showers averaged over all charged particle tracks.

\subsection{Light propagation and OM response}

Response of OM on a shower Cherenkov radiation is simulated in two steps. In first step the propagation of the Cherenkov radiation of point-like shower from the source to the photo-sensor is simulated taking into account the angular distribution of radiation in source and the spectral dependences of absorption and scattering of light in water, PMT quantum efficiency as well as 
Cherenkov light intensity and light velocity. A volume about of $10^{8} \mathrm{~m}^{3}$ around a shower origin is filled by detection spheres with a size of OM. When a simulated photon, moving in direction $\Omega(\theta, \varphi)$, crosses the detection sphere located at the distance $\mathrm{r}(\rho, \mathrm{z})$ from the shower vertex in a time $t$ the value in a corresponding cell of table, which describes the photon spatial, direction and temporal distribution, is increased on the value of PMT quantum efficiency corresponding to photon wavelength. At the second step a five-dimensional table for the expected average number of photoelectrons $n_{p e}(\rho, z, \theta, \varphi, \tau)$ is generated by folding the photon flux obtained in first step with $\mathrm{OM}$ angular sensitivity. The variables $\rho$ and $z$ characterize the OM position relative to a point-like shower - the distance from the OM to the shower axis $\rho$ and the coordinate along the $z$ axis oriented in the direction opposite to that of the shower axis. The polar angle $\theta$ (measured from the direction coincident with the $z$ direction) and the azimuth angle $\varphi$ characterize the orientation of the $\mathrm{OM}$ with respect to the shower. The variable $\tau$ characterizes the time delay of the recorded photons relative to the arrival time of the direct photons.

\subsection{Reconstruction method}

The procedure for reconstructing the parameters of high-energy showers - the shower energy, direction, and vertex - is performed in two steps. In the first step, the shower vertex coordinates are reconstructed using the time information from the telescope's triggered photosensors. In this case, the shower is assumed to be a point-like source of light. The $\chi^{2}$ minimization parameters are shower coordinates $((\mathrm{x}, \mathrm{y}, \mathrm{z})$ in a cartesian coordinate system or $(r, \theta, \varphi)$ in a spherical coordinate system):

$$
\chi_{t}^{2}=\frac{1}{\left(N_{\text {hit }}-4\right)} \sum_{i=1}^{N_{\text {hit }}} \frac{\left(T_{i}\left(\vec{r}_{s h}, t_{0}\right)-t_{i}\right)^{2}}{\sigma_{t i}^{2}}
$$

where $t_{i}$ and $T_{i}$ are the measured and theoretically expected trigger times of the $i$ th photo-sensor, $t_{0}$ - the shower generation time, $\sigma_{t i}$ - the uncertainty in measuring the time, and $N_{h i t}$ is the hit multiplicity. In the case of detecting the Cherenkov radiation of high-energy showers in the Baikal water, the bulk of the photo-sensors are triggered from direct photons or those scattered through small angles. This simplifies considerably the shower coordinate reconstruction procedure. More specifically, the propagation time of the direct photons from the shower to the corresponding photo-sensor can be chosen as the theoretically expected time $T_{i}$. The reconstruction quality can be increased by applying additional event selection criteria based on the limitation of the admissible values for the specially chosen parameters characterizing the events.

In the second step, the shower energy and direction are reconstructed by applying the maximum-likelihood method and using the shower coordinates reconstructed in the first step. The values of the variables $\theta, \varphi$, and $E_{s h}$ corresponding to the minimum value of the following functional are chosen as the polar and azimuth angles characterizing the direction and the shower energy:

$$
L_{A}=-\sum_{i=1}^{N_{\text {hit }}} \ln p_{i}\left(A_{i}, E_{s h}, \vec{\Omega}_{s h}(\theta, \varphi)\right) .
$$

The functions $p_{\mathrm{i}}\left(\mathrm{A}_{\mathrm{i}}, \mathrm{E}_{\mathrm{sh}}, \Omega_{\mathrm{sh}}(\theta, \varphi)\right)$ are the probabilities for a signal with amplitude $A_{i}$ (measured in photoelectrons) from a shower with energy $E_{s h}$ and direction $\Omega_{s h}$ to be recorded by the $i$ th triggered photo-sensor:

$$
p_{i}=\sum_{n=1}^{\infty} P(n / \bar{n}) \int_{A_{i}-\frac{\alpha}{2}}^{A_{i}+\frac{\alpha}{2}} \xi_{i}(A, n) d A,
$$


where $P(n / \bar{n})$ is the probability of detecting $n$ photoelectrons at a mean $\bar{n}$ for the Poisson distribution, $\xi(A, n)$ is the probability density function for recording the amplitude $A$ at an exposure level of $n$ photoelectrons, and $\alpha$ is the scale-division value of the amplitude in photoelectrons. The mean $\bar{n}$ are determined by simulating the responses of the telescope's OMs to the Cherenkov radiation of a shower with energy $E_{6 h}$ and direction $\Omega_{s h}$ with allowance made for the light propagation in water, the relative positions and orientation of the OMs and the shower, and the effective OM sensitivity.

\subsection{Response of array on showers induced by neutrinos}

Vertex of shower is randomly generated in a sufficiently large rectangular box around an array. Then the propagation of neutrino with initial energy $E_{v}$ from the Earth surface to point of shower origin is simulated taking into account $\mathrm{CC}$ and $\mathrm{NC}$ processes. For $v_{\mathrm{e}}$ and $v_{\mu}$ only points of $\mathrm{NC}$ interactions are generated and new neutrino energy is calculated. Neutrino absorption due to $\mathrm{CC}$ interaction on the pass length $l$ is taken into account by $\exp \left(-l / l^{c c}\right)$ weight. In the case of $v_{\tau}$ the points of both $\mathrm{CC}$ and $\mathrm{NC}$ interactions are generated. For CC interaction, the $\tau$ decay mod is simulated and propagation process of the secondary neutrinos is continued. In the point of the shower vertex the final energy of neutrino is obtained and shower energy is generated due to CC or NC interactions. In the next step, the responses of optical modules are simulated using the response table described above. Finally, the fulfilment of trigger conditions is tested. An effective area and effective volume are calculated in conventional way: $S=S_{0} \frac{N_{t r}}{N_{0}}$ and $V=V_{0} \frac{N_{t r}}{N_{0}}$ where $\mathrm{N}_{0}$ and $\mathrm{N}_{\text {tr }}$ are generated and detected number events, $\mathrm{V}_{0}-$ volume of the rectangular box and $\mathrm{S}_{0}-$ cross section of box for neutrino direction.

\subsection{GVD cluster "Dubna"}

In April 2015 the first cluster of Baikal-GVD was deployed and started operation in Lake Baikal. The array operation time matched 213 days from April 2015 till February 2016. This array was named "Dubna". It encloses several Megatons of the fresh waters of Lake Baikal. The first cluster of Baikal-GVD comprises a total of 192 optical modules arranged at eight $345 \mathrm{~m}$ long strings, as well as an acoustic positioning system and an instrumentation string with equipment for array calibration and monitoring of environmental parameters. Each string comprises $24 \mathrm{OMs}$ spaced by $15 \mathrm{~m}$ at depths of $900 \mathrm{~m}$ to $1250 \mathrm{~m}$ below the surface. In 2015 seven side strings have been located at a reduced radius of $40 \mathrm{~m}$ around a central one (compared to $60 \mathrm{~m}$ for the baseline configuration). The reason is to increase the sensitivity to low-energy atmospheric muons and neutrinos which are used for array calibration. In the 2016, strings have been moved to the baseline distances.

The search for high-energy neutrinos with "Dubna" array is based on the selection of cascade events generated by neutrino interactions in the sensitive volume of array. Performances of event selection and cascade reconstruction procedures were tested by MC simulation of signal and background events and reconstruction parameters of cascades. After reconstruction of cascade vertex, energy and direction and applying quality cuts, events with a final multiplicity of hit OMs $\mathrm{N}_{\text {hit }}>20$ were selected as high-energy neutrino events. Accuracy of cascade energy reconstruction is about $30 \%$, the accuracy of direction reconstruction is about 4 degree (median value) and vertex resolution is about $2 \mathrm{~m}$ [7]. Neutrino effective areas for different flavours averaged over all arrival angles are shown in Fig.2(left). Energy distributions of cascade events expected for one year observation from astrophysical fluxes following a power law $\mathrm{E}^{-2}$ and $\mathrm{E}^{-2.46}$ spectra and single-flavour normalizations $1.2 \times 10^{-8} \mathrm{GeV}^{-1} \mathrm{~cm}^{-2} \mathrm{~s}^{-1} \mathrm{sr}^{-1}$ and $4.1 \times 10^{-6} \mathrm{GeV}^{-1} \mathrm{~cm}^{-2} \mathrm{~s}^{-1} \mathrm{sr}^{-1} \mathrm{re}-$ spectively $[6,8]$, as well as distribution of expected background shower events from atmospheric 
neutrinos are shown in Fig. 2 (right). The expected number of background events from atmospheric neutrinos is strongly suppressed for energies higher than $100 \mathrm{TeV}$. About 0.5 and 0.4 cascade events per year with energies above $100 \mathrm{TeV}$ and hit multiplicities $\mathrm{N}_{\text {hit }}>20$ from astrophysical fluxes with $\mathrm{E}^{-2}$ and $\mathrm{E}^{-2.46}$ spectra respectively and 0.08 background events from atmospheric neutrinos are expected.
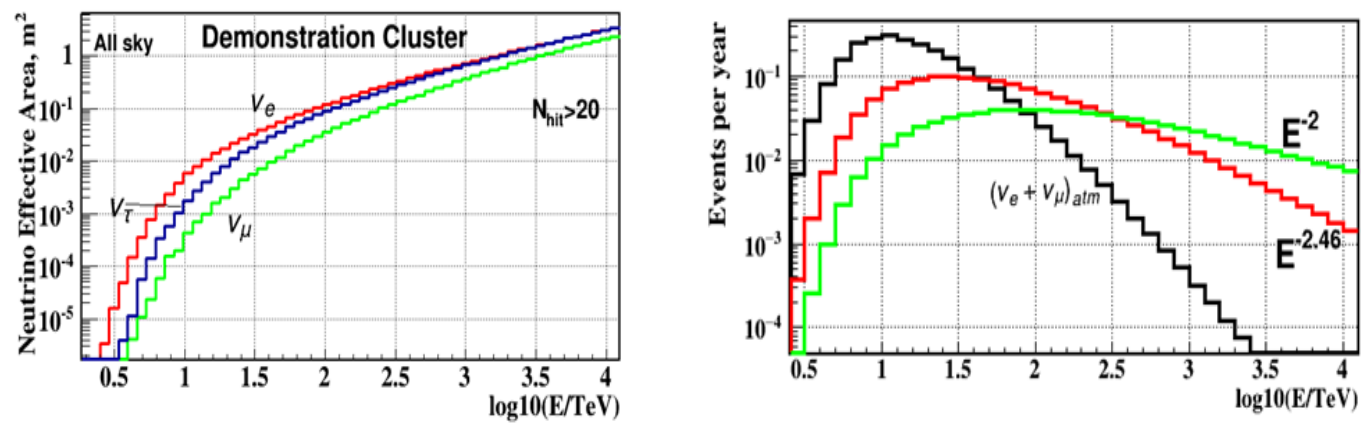

Fig. 2: Left: Neutrino effective areas for different flavours averaged over all arrival angles. Right: Energy distributions of events expected for one year observation from astrophysical fluxes with $E^{-2}$ and $E^{-2.46}$ spectra and IceCube normalization (see text). Also shown is a distribution of expected background events from atmospheric neutrinos.

\section{Results}

For search for high-energy neutrino flux of astrophysical origin the data collected from 24 October till 17 December 2015 have been used. A data sample of $4.4 \times 10^{8}$ events has been accumulated by array trigger, which corresponds to 41.64 array live days. Causality cuts and requirement of $\mathrm{N}>3$ hit OMs rest about $1.8 \times 10^{7}$ events for following analysis. After applying an iterative procedure of cascade vertex reconstruction for hits with charge higher 1.5 ph.el., followed by the rejection of hits contradicting the cascade hypothesis on each iteration stage, 316229 events survived as cascade-like events. After cascade energy reconstruction and event quality cuts applying, 12931 cascade-like events have been selected. Hit OM multiplicity distribution of these events is shown in Fig.3(left), as well as the expected similar distribution of background events from atmospheric muons. Total of 1192 events from final sample were reconstructed with energies above $100 \mathrm{TeV}$. Hit $\mathrm{OM}$ multiplicity distribution of these events is shown in Fig.3 (right). Also shown are expected event distributions from astrophysical flux with $\mathrm{E}^{-2.46}$ spectrum and IceCube normalization, as well as distributions from atmospheric muons and atmospheric neutrinos. Statistics of generated atmospheric muon sample relates to 72 live days of data taking.
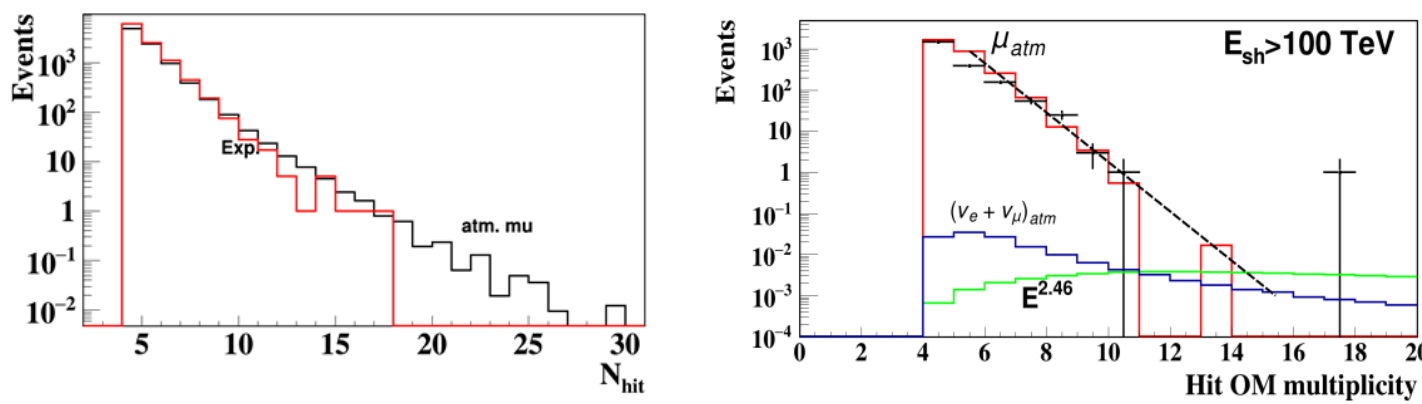

Fig. 3: Left: Hit OM multiplicity distribution of experimental events after all cuts. Also, similar distribution expected from atmospheric muons is shown. Right: Hit OM multiplicity distribution of experimental events with reconstructed energy $\mathrm{E}_{\mathrm{rec}}>100 \mathrm{TeV}$ (dots). Also shown are distributions of expected events from astrophysical neutrinos with $\mathrm{E}^{-2.46}$ spectrum and background events from atmospheric muons and neutrinos. 
All of experimental events, but one, have hit OM multiplicities less than 10 OMs and are consistent with expected background events from atmospheric muons. One event with 17 hit OMs was reconstructed as downward moving cascade. For more precise reconstruction of cascade parameters, this event was reanalysed including hits with amplitudes lower 1.5 ph.el.. Total of 24 hits are consistent with a cascade hypothesis and reconstructed cascade parameters are following: cascade energy $\mathrm{E}=107 \mathrm{TeV}$, zenith angle $\theta=59^{\circ}$ and azimuthal angle $\varphi=130.5^{\circ}$, distance from array axis $\rho=68 \mathrm{~m}$.

\section{Conclusion}

The first, demonstration cluster of the Baikal-GVD neutrino telescope was taking data during 2015. A search for high-energy cascad-like events has been performed using a data sample corresponding to 41 live-days of array operation. Total of 1191 events were selected as cascade events with hit multiplicity $\mathrm{N}_{\text {hit }}<10$. This result is consistent with expectation from atmospheric muons. One selected event has high hit multiplicity $\mathrm{N}_{\text {hit }}$ $=17$ (hits with charges above a threshold of 1.5 ph.el.). Results obtained with this analysis demonstrate high quality of data, as well as efficiency of analysis procedures. The data collected with Baikal-GVD during 2016 - 2017 years will substantially increase sensitivity to neutrino fluxes of different origin.

This work was supported by the Russian Foundation for Basic Research (Grants 16-2913032, 17-02-01237).

\section{References}

[1] V. Aynutdinov et al., The prototyping/early construction phase of the BAIKAL-GVD project. NIM A742 82-88 (2014).

[2] M. G. Aartsen et al., IceCube Coll., Evidence for high-energy extraterrestrial neutrinos at the IceCube detector. Science 342, 1242856 (2013), [arXiv:1311.5238 [astro-ph.HE]].

[3] C. Kopper, W. Giang and N. Kurahashi for the IceCube Coll., in Proc. 34th Int. Cosmic Ray Conference, Vol. 001, 1081. PoS, 2015.

[4] V. Aynutdinov et al., Astropart.Phys. 25, 140 (2006).

[5] A. Avrorin et al., Astronomy Letters 35, 651 (2009).

[6] M.G.Aartsen et al. , Phys. Rev. Lett. 113101101.

[7] A.D.Avrorin et al., Phys.Part.Nucl. 46 no.2, 211-221 (2015) .

[8] M. G. Aartsen et al., IceCube Coll., Phys. Rev. D 91, 022001 (2015). 